\title{
Effect of Nutrient Management and Crop Geometry in Sweet Flag
}

\author{
C. Payeng* , A. Sharma and B. Gogoi \\ Department of Agronomy, Assam Agricultural University, Jorhat, Assam-785013, India \\ *Corresponding author
}

\begin{tabular}{l} 
Ke y w o r d s \\
$\begin{array}{l}\text { Acorus calamus, } \\
\text { Benefit: cost ration, } \\
\text { Nutrient } \\
\text { management }\end{array}$ \\
\hline Article Info \\
$\begin{array}{l}\text { Accepted: } \\
\text { 20 May } 2018 \\
\text { Available Online: } \\
\text { 10 June } 2018\end{array}$ \\
\hline
\end{tabular}

A B S T R A C T

A field experiment entitled "Effect of nutrient management and crop geometry in sweet flag (Acorus calamus)" was carried out at Instructional-cum-Research farm, Assam Agricultural University, Jorhat during 2012-2013 in randomized block design (RBD) with three replications to assess the effect of different crop geometry and application of different organic and inorganic fertilizers on plant growth and development and yield attributing characters for commercial production. The results revealed that wider crop spacing $(30 \mathrm{~cm} \times 30 \mathrm{~cm})$ has significantly higher growth i.e., plant height and dry matter and yield attributing characters i.e., rhizome weight, size of rhizome (length and girth) and number of plants/clump than close spacing. However, closer spacing recorded significantly higher yield (rhizome and oil) and N, P, K uptake as well as higher gross return (Rs.107583.00), net return (Rs.63879.00) and benefit: cost ratio (1.45) than wider spacing. Application of 20-10-15 N- $\mathrm{P}_{2} \mathrm{O}_{2}-\mathrm{K}_{2} \mathrm{O} \mathrm{kg} / \mathrm{ha}+$ Vermicompost @ $1 \mathrm{t} / \mathrm{ha}$ being at par with 60-30-45 N-P $\mathrm{P}_{2}-\mathrm{K}_{2} \mathrm{O} \mathrm{kg} / \mathrm{ha}$ recorded significantly higher growth, yield attributes, yield and N, P, K uptake of Acorus calamus. Application of 20-10-15 N- $\mathrm{P}_{2} \mathrm{O}_{2}-\mathrm{K}_{2} \mathrm{O} \mathrm{kg} / \mathrm{ha}+$ Vermicompost @ 1 t/ha recorded the highest gross return (Rs.104500.00) followed by 6030-45 N-P $\mathrm{O}_{2}-\mathrm{K}_{2} \mathrm{O} \mathrm{kg} / \mathrm{ha}$. However 60-30-45 N- $\mathrm{P}_{2} \mathrm{O}_{2}-\mathrm{K}_{2} \mathrm{O} \mathrm{kg} / \mathrm{ha}$ recorded the highest net return (Rs.65911.00) followed by 20-10-15 N- $\mathrm{P}_{2} \mathrm{O}_{2}-\mathrm{K}_{2} \mathrm{O} \mathrm{kg} / \mathrm{ha}+$ Vermicompost @ $1 \mathrm{t} / \mathrm{ha}$ (Rs. 60617.00).

\section{Introduction}

Sweet flag (Acorus calamus) is a semi aquatic perennial rhizomatous monocot herb of the family Acoraceae that usually grows in ponds, lakes, marshes and in the bank of quiet waters along with other aquatic weeds.

It is commonly known as Bach in India. The leaves are yellow green in colour, sword shaped, thick textured, and from $75-100 \mathrm{~cm}$ in length. The stem is modified to rhizome with a diameter of $3-4 \mathrm{~cm}$ which is elongated that runs across the ground, and possesses a pungent, bitter taste when fresh and milder when dried. The rhizome is marked by leaf scars above, and produces abundant roots, which for the most part go as straight down, below.

The scented leaves and rhizomes have been traditionally used medicinally against different ailments like fever, asthma, bronchitis, cough and mainly for digestive problems such as gas, bloating, colic and poor digestive function for at least 2000 years. 
The rhizome is the source of essential oil having the antibacterial, antifungal and insecticidal properties. Now a days rhizome oil of Acorus calamus is widely used in pharmaceuticals and traditional systems of medicines for a number of ailments and in perfumes. Acorus oil and the raw material, originated from the natural wild populations is a common medicinal item in the international drug market.

Because of the medicinal potential, the herb has been overexploited rapidly from the natural wild habitats and depleted its population during the last three decades and the herb is placed under threatened category.

Therefore, to satisfy the demand of the raw materials in the market, the herb needs an immediate conservation of genetic resources as well as its field cultivation. It is reported that Acorus calamusis cultivated throughout India, up to altitudes of about 2200m (McAlpine \& Warrier Limited, 1996). To have a better crop stand and productivity, adoption of proper agro technology is very important.

Acorus calamus is a annual long-day plant with life cycle of one year in general. Crop geometry is one of the important growth parameter which influences on the favorable yield attributes (Tiwari, 2004). Crop geometry also affects the growth and yield of Acorus calamus rhizome (Singh and Nongmaithem, 2013). Therefore, to have a better productivity of rhizome, adoption of proper crop geometry is very necessary as the rhizome is the main important part of the herb.

Soil nutrient always takes an important part in the growth, development and yield of plant. Nitrogen is a major part of the chlorophyll molecule and is therefore necessary for photosynthesis. Phosphorus plays a major role in energy storage and transfer as ADP and ATP and thus plays an important role in photosynthesis as well as respiration. Presence of potassium plays a vital role in plant growth because $\mathrm{K}$ is known to be an enzyme activator that promotes metabolism. Acorus calamus responded well to higher doses of nitrogen (Tiwari, 2000). Also it is reported that availability micro and macro nutrients increase the productivity of the herb (Datta, 2009).

Maintenance of soil fertility is a prerequisite for long-term sustainable agriculture where organic manure can play a vital role in the sustenance of soil fertility and crop production (Jablonska, 1990). Vermicompost is suitable organic manure and it contains all the essential plant nutrients. It contains $1.5-2.5 \%$ Nitrogen, 0.9-1.7\% Phosphorus and 1.5-2.4\% Potash which is very high as compared to other organic manures. Though vermicompost has high nutrient content it is not possible to meet the entire nutritional requirements from the vermicompost only to have higher productivity. Besides, due to slow release of plant nutrients from organic manure, the potentialities of organic source is very limited to afford higher crop production. To overcome this problem, application of organic manures in conjunction with inorganic fertilizers, called integrated nutrient management, can play important role in cultivation of crop.

The present experiment was, therefore, undertaken to study the performance of Acorus calamus under different crop geometry and levels of nutrient with the following objectives:

To find the optimum spacing of sweet flag

To study the effect of organic and inorganic nutrient sources on growth and yield of sweet flag

To find out the suitable dose of nutrient for commercial production 


\section{Materials and Methods}

The field experiment was conducted in Instructional-cum-Research (ICR) Farm, Assam Agricultural University, Jorhat-13. The soil texture was clay loam having available $\mathrm{N}$, $\mathrm{P}_{2} \mathrm{O}_{5}$, and $\mathrm{K}_{2} \mathrm{O} 262.3,27.2$ and $213.0 \mathrm{~kg} / \mathrm{ha}$ respectively with $\mathrm{pH} 5.48$ and organic carbon 0.71.The experiment was laid out in Factorial randomized block design with 3 replications having 12 plots (plot size $4 \mathrm{~m} \times 3 \mathrm{~m}$ ) in each replication. The treatments consisted of Two Crop Geometry viz, $20 \mathrm{~cm} \times 20 \mathrm{~cm}\left(\mathrm{~S}_{1}\right)$ and $30 \mathrm{~cm} \times 30 \mathrm{~cm}\left(\mathrm{~S}_{2}\right)$ and six level of nutrient management viz., $\mathrm{T}_{1}$ [20-10-15 N-P $\mathrm{O}_{5}-$ $\left.\mathrm{K}_{2} \mathrm{O} \quad \mathrm{kg} / \mathrm{ha}\right], \mathrm{T}_{2}\left[40-20-30 \quad \mathrm{~N}-\mathrm{P}_{2} \mathrm{O}_{5}-\mathrm{K}_{2} \mathrm{O}\right.$ $\mathrm{kg} / \mathrm{ha}$ ], $\mathrm{T}_{3}\left[60-30-45 \mathrm{~N}-\mathrm{P}_{2} \mathrm{O}_{5}-\mathrm{K}_{2} \mathrm{O} \mathrm{kg} / \mathrm{ha}\right]$, $\mathrm{T}_{4}$ [Vermicompost @2 t/ha], T 5 [20-10-15 N$\mathrm{P}_{2} \mathrm{O}_{5}-\mathrm{K}_{2} \mathrm{O} \mathrm{kg} / \mathrm{ha}+$ Vermicompost @ $\left.1 \mathrm{t} / \mathrm{ha}\right]$ and $\mathrm{T}_{6}[\mathrm{Control}]$.

Land was harrowed before monsoon rain. After receiving monsoon rain, the land was puddled with cultivator twice before planting. Previous year's rhizome pieces with active buds of local variety were used as planting materials. Rhizomes were sown in puddle nursery bed. Sprouted rhizomes of 2-3 cm length with attached leaves were selected and transplanted manually by placing $4-5 \mathrm{~cm}$ depth at crop geometry as per treatment required that is $20 \mathrm{~cm} \times 20 \mathrm{~cm}$ and $30 \mathrm{~cm} \times 30 \mathrm{~cm}$. Recommended levels of nutrients were applied before transplanting the crop as per the treatments. $1 / 3^{\text {rd }}$ of $\mathrm{N}$ and full doses $\mathrm{P}_{2} \mathrm{O}_{5}$ and $\mathrm{K}_{2} \mathrm{O}$ were applied as basal in the form of urea, single super phosphate (SSP) and muriate of potash (MOP), respectively. $1 / 3^{\text {rd }}$ was applied one and two months after planting. Vermicompost (2t/ha) was used as substitute of chemical fertilizer and was applied as basal before transplanting. During the growing period plants morphological characters and growth i.e., Plant height, Dry matter Accumulation and Partitioning, Leaf Area Index, Crop Growth Rate were studied at
30, 60, 90, 120 and 150 DAT and at harvest of the crop. Yield attributing characters viz. Plant population, Numbers of plant/clump, Size of Rhizome (Length and Girth) were carried out at harvesting.

Plants with rhizome were dug out manually with the help of hoe and dried in sun. The weights of leaf as well as rhizome of the plants were taken separately in $\mathrm{kg} / \mathrm{plot}$ and then converted to $\mathrm{kg} / \mathrm{ha}$ and recorded for each plot. Samples i.e., leaf and rhizomes from each plot were taken for further laboratory analysis.

Grinded rhizome samples of $10 \mathrm{~g}$ each from all the treatments were taken for extraction of oil. The crushed sample was placed in a themble and extracted with light petroleum ether in a soxhelt extraction unit as per method described by AOAC (1980). The extract was transferred to a weighed flask, the solvent was distilled of and the last traces of solvent and moisture being removed by heating the flask at $100-150^{\circ} \mathrm{C}$. Then the flask was collected and reweighed.

The plant samples (both leaf and rhizome) collected at harvest for each plot were dried in oven at $60^{\circ} \mathrm{C}$. The oven-dried samples were ground to powder and analysed for $\mathrm{N}, \mathrm{P}$ and $\mathrm{K}$ (\%).Nitrogen content in leaf and rhizome was estimated by modified Kjeldahl method as described by Jackson (1973).Phosphorus content in leaf and rhizome were determined colorimetrically by tri acid digestion and yellow colour method as outlined by Jackson (1973).Potassium content in leaf and rhizome were determined by flame photometer as described by Jackson (1973).

Likewise soil samples collected from each plot were air-dried, ground and sieved through 2 $\mathrm{mm}$ diameter sieve and were used for estimation of available nitrogen, phosphorus, potassium organic carbon bulk densityand $\mathrm{pH}$. 
Available nitrogen was estimated by Modified Kjeldahl method and expressed in $\mathrm{kg} / \mathrm{ha}$. Available phosphorus was estimated by Bray I method and Potassium was estimated by Flame photometric method. Organic cabron was estimated by Walkley and Black's method.pH of the soil solution was estimated by "1:25 (soil: water) suspension glass electrode $\mathrm{pH}$ meter" method as described by Jackson (1973).

All the data pertaining to the present investigation wherever needed were statistically analyzed for randomized block design (RBD) described by Panse and Sukhatme (1985). Critical differences (CD) at $5 \%$ probability level was calculated only when the $\mathrm{F}$ value has been found to be significant.

\section{Results and Discussion}

The results of the present investigation conducted on "Effect of nutrient management and crop geometry in sweet flag (Acorus calamus)" presented in the previous chapter revealed the effect of different treatments on various growth and yield attributing characters of the plant. In this chapter, the reasons for variation in various parameters and results are discussed and explained.

\section{Growth and Yield attributing characters}

In the present study, crop geometry did not bring about any significant variation in crop growth at early stage of observations (up to 30 DAT). The growth of Acorus calamus measured in terms of plant height, total dry weight as well as dry matter accumulation in leaves and rhizome varied significantly under different crop geometry at different stages of observation. Crop geometry $30 \mathrm{~cm} \times 30 \mathrm{~cm}$ recorded higher biomass accumulation in different plant parts as well as total biomass than that at crop geometry $20 \mathrm{~cm} \times 20 \mathrm{~cm}$. The better growth under the wider crop geometry might be due to the less intra specific competition as compared to closure crop geometry.Singh and Nongmaithem (2013) also observed improvement in overall growth of Acorus calamus under wider crop geometry. Growth in terms of crop growth rate and leaf area index was observed to be higher atcloser spacing of $20 \mathrm{~cm} \times 20 \mathrm{~cm}$ and this could be due to higher plant density resulting in dry matter accumulation per unit area. Results of almost similar nature have been reported by Arbha et al., (2014) in potato.

Among the different nutrient management practices, application of both organic and inorganic fertilizer i.e., $20-10-15 \mathrm{~kg} / \mathrm{ha} \mathrm{N}$ $\mathrm{P}_{2} \mathrm{O}_{5}-\mathrm{K}_{2} \mathrm{O}+$ Vermicompost @ $1 \mathrm{t} / \mathrm{ha}$ recorded an increase in plant height from the the day of first sampling (30DAT) till harvest and was follwed by the application of $60-30-45 \mathrm{~kg} / \mathrm{ha}$ $\mathrm{N}-\mathrm{P}_{2} \mathrm{O}_{5}-\mathrm{K}_{2} \mathrm{O}$. Other growth characters like total dry weight, dry matter accumulation, crop growth rate and leaf area index was also found to increase with combined application of both organic and inorganic fertilizer i.e., 20-10-15 kg/ha N- $\mathrm{P}_{2} \mathrm{O}_{5}-\mathrm{K}_{2} \mathrm{O}+$ Vermicompost $(1 \mathrm{t} / \mathrm{ha})$ and was at par with application of 60$30-45 \mathrm{~kg} / \mathrm{ha} \mathrm{N}-\mathrm{P}_{2} \mathrm{O}_{5}-\mathrm{K}_{2} \mathrm{O}$.

Application of organic and inorganic fertilizers increased the availability of both macro and micro plant nutrients throughout the growth period resulting higher yield attributing characters. Results of almost similar nature have been reported by Datta et al., (2009) and Singh et al., (2010).

The yield attributing characters like number of plant per clump, rhizome weight, size of rhizome (length and girth) per plant increased significantly under wider crop spacing of 30 $\mathrm{cm} \times 30 \mathrm{~cm}$. Wider spacing led to less intra plant competition and because of it higher yield attributing characters were experienced in the present study. Similar results were experienced by Tiwari et al., (2012). 
Table.1 Effect of different treatments on plant height and dry matter accumulation

\begin{tabular}{|c|c|c|c|c|c|c|c|c|c|c|c|}
\hline \multirow[t]{2}{*}{ Treatment } & \multicolumn{5}{|c|}{ Plant height (cm) } & \multicolumn{6}{|c|}{ Dry matter (g) } \\
\hline & $\begin{array}{c}30 \\
\text { DAP }\end{array}$ & $\begin{array}{c}60 \\
\text { DAP }\end{array}$ & 90 DAP & $\begin{array}{l}120 \\
\text { DAP }\end{array}$ & $\begin{array}{c}150 \\
\text { DAP }\end{array}$ & 30DAP & 60DAP & 90DAP & $\begin{array}{c}120 \\
\text { DAP }\end{array}$ & $\begin{array}{c}150 \\
\text { DAP }\end{array}$ & Harvest \\
\hline \multicolumn{12}{|l|}{ Crop Geometry (S) } \\
\hline $\mathrm{S}_{1}=20 \mathrm{~cm} \times 20 \mathrm{~cm}$ & 28.01 & 40.32 & 65.03 & 75.16 & 75.34 & 3.87 & 7.55 & 13.13 & 15.56 & 16.10 & 16.23 \\
\hline$S_{2}=30 \mathrm{~cm} \times 30 \mathrm{~cm}$ & 29.72 & 41.23 & 65.82 & 76.45 & 76.56 & 3.86 & 9.95 & 18.04 & 20.35 & 20.73 & 20.86 \\
\hline SEm \pm & 0.47 & 0.65 & 0.67 & 0.67 & 0.66 & 0.10 & 0.14 & 0.29 & 0.28 & 0.38 & 0.40 \\
\hline$C D(P=0.05)$ & NS & NS & NS & NS & NS & NS & 0.43 & 0.84 & 0.83 & 1.11 & 1.16 \\
\hline \multicolumn{12}{|l|}{ Nutrient levels (T) } \\
\hline $\mathbf{T}_{1}=20-10-15$ & 29.00 & 40.74 & 65.74 & 76.14 & 76.27 & 3.69 & 8.25 & 14.58 & 16.99 & 17.42 & 17.60 \\
\hline $\mathbf{T}_{2}=40-20-30$ & 29.22 & 42.07 & 67.04 & 77.44 & 77.56 & 3.81 & 9.26 & 16.48 & 18.95 & 19.32 & 19.49 \\
\hline $\mathbf{T}_{\mathbf{3}}=60-30-45$ & 29.43 & 42.46 & 67.46 & 78.03 & 78.11 & 4.10 & 9.84 & 17.48 & 20.07 & 20.55 & 20.72 \\
\hline $\begin{array}{l}\mathbf{T}_{4}=\text { Vermicompost } \\
@ 2 \mathrm{t} / \mathrm{ha}\end{array}$ & 29.11 & 41.43 & 66.53 & 76.93 & 77.13 & 3.82 & 8.42 & 15.06 & 17.33 & 17.76 & 17.92 \\
\hline $\begin{array}{l}\mathbf{T}_{5}=20-10-15+ \\
\text { Vermicompost @ } 1 \\
\text { t/ha }\end{array}$ & 30.14 & 43.11 & 68.13 & 78.71 & 78.84 & 4.60 & 10.00 & 17.75 & 20.38 & 20.87 & 21.03 \\
\hline $\mathrm{T}_{6}=$ Control & 26.91 & 35.03 & 57.71 & 67.44 & 67.56 & 3.19 & 6.71 & 12.13 & 14.02 & 14.55 & 14.55 \\
\hline SEm \pm & 0.82 & 1.13 & 1.17 & 1.17 & 1.14 & 0.18 & 0.25 & 0.50 & 0.49 & 0.66 & 0.69 \\
\hline $\mathrm{CD}(\mathrm{P}=0.05)$ & 2.41 & 3.31 & 3.43 & 3.40 & 3.32 & 3.87 & 7.55 & 13.13 & 15.56 & 16.10 & 16.23 \\
\hline Interaction (S x T) & NS & NS & NS & NS & NS & 3.86 & 9.95 & 18.04 & 20.35 & 20.73 & 20.86 \\
\hline
\end{tabular}


Table.2 Dry matter partitioning of Acorus calamus (g/plant) as influenced by different treatments at 30, 60, 90, 120, 150 and at Harvest

\begin{tabular}{|c|c|c|c|c|c|c|c|c|c|c|c|c|}
\hline \multirow[t]{3}{*}{ Treatment } & \multicolumn{12}{|c|}{ Dry weight (g) } \\
\hline & Leaf & $\begin{array}{l}\text { Rhiz } \\
\text { ome }\end{array}$ & Leaf & $\begin{array}{c}\text { Rhizom } \\
\text { e }\end{array}$ & Leaf & $\begin{array}{c}\text { Rhizom } \\
\text { e }\end{array}$ & Leaf & $\begin{array}{c}\text { Rhizom } \\
\text { e }\end{array}$ & Leaf & Rhizome & Leaf & $\begin{array}{c}\text { Rhizom } \\
\text { e } \\
\end{array}$ \\
\hline & \multicolumn{2}{|c|}{ 30DAP } & \multicolumn{2}{|c|}{ 60DAP } & \multicolumn{2}{|c|}{90 DAP } & \multicolumn{2}{|c|}{120 DAP } & \multicolumn{2}{|c|}{150 DAP } & \multicolumn{2}{|c|}{ Harvest } \\
\hline \multicolumn{13}{|l|}{ Crop Geometry (S) } \\
\hline$S_{1}=20 \mathrm{~cm} \times 20 \mathrm{~cm}$ & 2.11 & 1.76 & 1.76 & 3.14 & 7.39 & 5.72 & 7.35 & 8.21 & 6.04 & 10.05 & 4.50 & 11.73 \\
\hline$S_{2}=30 \mathrm{~cm} \times 30 \mathrm{~cm}$ & 2.19 & 1.83 & 1.83 & 4.15 & 10.50 & 8.11 & 10.78 & 9.57 & 6.80 & 13.93 & 5.80 & 15.06 \\
\hline SEm \pm & 0.04 & 0.05 & 0.05 & 0.05 & 0.18 & 0.16 & 0.13 & 0.11 & 0.17 & 0.12 & 0.10 & 0.28 \\
\hline $\mathrm{CD}(\mathrm{P}=0.05)$ & NS & NS & NS & 0.14 & 0.52 & 0.47 & 0.37 & 0.32 & 0.49 & 0.36 & 0.30 & 0.82 \\
\hline \multicolumn{13}{|l|}{ Nutrient levels (T) } \\
\hline $\mathbf{T}_{1}=20-10-15$ & 2.02 & 1.68 & 1.68 & 3.44 & 9.09 & 7.16 & 8.58 & 8.41 & 5.97 & 11.46 & 4.88 & 12.72 \\
\hline $\mathbf{T}_{\mathbf{2}}=40-20-30$ & 2.31 & 1.92 & 1.92 & 3.86 & 9.32 & 7.17 & 9.57 & 9.38 & 6.78 & 12.54 & 5.47 & 14.02 \\
\hline $\mathbf{T}_{3}=60-30-45$ & 2.45 & 2.04 & 2.04 & 4.10 & 9.88 & 7.60 & 10.14 & 9.94 & 7.39 & 13.16 & 5.81 & 14.90 \\
\hline $\begin{array}{l}\mathbf{T}_{4}=\text { Vermicompost @ } \\
2 \text { t/ha }\end{array}$ & 2.06 & 1.71 & 1.71 & 3.51 & 8.51 & 6.55 & 8.75 & 8.58 & 6.11 & 11.65 & 4.96 & 12.96 \\
\hline $\begin{array}{l}\mathbf{T}_{\mathbf{5}}=20-10-15+ \\
\text { Vermicompost @1 } \\
\text { t/ha }\end{array}$ & 2.51 & 2.09 & 2.09 & 4.17 & 10.03 & 7.72 & 10.29 & 10.09 & 7.53 & 13.33 & 5.90 & 15.13 \\
\hline $\mathbf{T}_{6}=$ Control & 1.57 & 1.31 & 1.31 & 2.80 & 6.86 & 5.27 & 7.08 & 6.94 & 4.74 & 9.81 & 3.92 & 10.63 \\
\hline SEm \pm & 0.07 & 0.05 & 0.05 & 0.09 & 0.31 & 0.28 & 0.22 & 0.19 & 0.29 & 0.21 & 0.18 & 0.49 \\
\hline $\mathrm{CD}(\mathrm{P}=0.05)$ & 0.19 & 0.15 & 0.15 & 0.25 & 0.91 & 0.82 & 0.64 & 0.55 & 0.85 & 0.63 & 0.53 & 1.43 \\
\hline Interaction (S x T) & NS & NS & NS & NS & NS & NS & NS & NS & NS & NS & NS & NS \\
\hline
\end{tabular}


Table.3 Effect of different treatments on LAI and Other Yield attributing characters

\begin{tabular}{|c|c|c|c|c|c|c|c|c|c|c|}
\hline \multirow[t]{2}{*}{ Treatment } & \multicolumn{6}{|c|}{ LAI } & \multirow{2}{*}{$\begin{array}{c}\text { Plant } \\
\text { populati } \\
\text { on } / \mathrm{m}^{2}\end{array}$} & \multirow{3}{*}{$\begin{array}{c}\text { Number of } \\
\text { plant per } \\
\text { clump }\end{array}$} & \multirow{2}{*}{$\begin{array}{l}\text { Rhizome } \\
\text { length } \\
\text { (cm) }\end{array}$} & \multirow{2}{*}{$\begin{array}{l}\text { Rhizome } \\
\text { girth } \\
\text { (cm) }\end{array}$} \\
\hline & 30DAP & 60DAP & 90DAP & $\begin{array}{c}120 \\
\text { DAP }\end{array}$ & $\begin{array}{c}150 \\
\text { DAP }\end{array}$ & Harvest & & & & \\
\hline \multicolumn{10}{|l|}{ Crop Geometry (S) } & \\
\hline $\mathrm{S}_{1}=20 \mathrm{~cm} \times 20 \mathrm{~cm}$ & 1.06 & 1.54 & 2.36 & 3.23 & 3.37 & 2.39 & 104.44 & 4.22 & 50.44 & 5.72 \\
\hline$S_{2}=30 \mathrm{~cm} \times 30 \mathrm{~cm}$ & 0.49 & 0.90 & 1.50 & 2.11 & 2.20 & 1.56 & 75.30 & 6.78 & 55.94 & 6.30 \\
\hline SEm \pm & 0.01 & 0.02 & 0.04 & 0.05 & 0.06 & 0.04 & 1.87 & 0.12 & 0.63 & 0.08 \\
\hline $\mathrm{CD}(\mathrm{P}=0.05)$ & 0.04 & 0.06 & 0.11 & 0.13 & 0.17 & 0.11 & 5.46 & 0.34 & 1.84 & 0.23 \\
\hline \multicolumn{11}{|l|}{ Nutrient levels (T) } \\
\hline $\mathbf{T}_{1}=20-10-15$ & 0.73 & 1.16 & 1.83 & 2.54 & 2.65 & 1.88 & 84.26 & 5.08 & 51.00 & 5.86 \\
\hline $\mathbf{T}_{2}=40-20-30$ & 0.81 & 1.27 & 2.00 & 2.75 & 2.88 & 2.04 & 89.81 & 5.58 & 53.50 & 6.02 \\
\hline $\mathbf{T}_{3}=60-30-45$ & 0.87 & 1.38 & 2.17 & 2.98 & 3.12 & 2.21 & 99.07 & 6.00 & 56.00 & 6.23 \\
\hline $\begin{array}{l}\mathbf{T}_{4}=\text { Vermicompost @ } 2 \\
\text { t/ha }\end{array}$ & 0.74 & 1.18 & 1.87 & 2.58 & 2.69 & 1.91 & 89.53 & 5.50 & 52.50 & 5.97 \\
\hline $\begin{array}{l}\mathbf{T}_{\mathbf{5}}=20-10-15+ \\
\text { Vermicompost @1t/ha }\end{array}$ & 0.90 & 1.40 & 2.20 & 3.04 & 3.17 & 2.25 & 99.26 & 6.17 & 56.83 & 6.44 \\
\hline $\mathbf{T}_{6}=$ Control & 0.58 & 0.95 & 1.52 & 2.11 & 2.20 & 1.56 & 77.31 & 4.67 & 49.33 & 5.55 \\
\hline SEm \pm & 0.02 & 0.04 & 0.07 & 0.08 & 0.10 & 0.07 & 3.23 & 0.20 & 1.10 & 0.14 \\
\hline$C D(P=0.05)$ & 0.07 & 0.11 & 0.19 & 0.23 & 0.29 & 0.19 & 9.43 & 0.58 & 3.21 & 0.42 \\
\hline Interaction (S x T) & NS & NS & NS & NS & NS & NS & NS & NS & NS & NS \\
\hline
\end{tabular}


Table.4 Effect of different treatments on Rhizome yield and oil content

\begin{tabular}{|c|c|c|c|c|}
\hline Treatment & Fresh weight (t/ha) & Dry weight (t/ha) & $\%$ Oil content & $\begin{array}{c}\text { Oil Yield } \\
\text { (kg/ha) }\end{array}$ \\
\hline \multicolumn{5}{|l|}{ Crop Geometry (S) } \\
\hline $\mathrm{S}_{1}=20 \mathrm{~cm} \times 20 \mathrm{~cm}$ & 8.50 & 2.15 & 5.35 & 115.16 \\
\hline$S_{2}=30 \mathrm{~cm} \times 30 \mathrm{~cm}$ & 6.06 & 1.53 & 5.33 & 81.93 \\
\hline SEm \pm & 0.17 & 0.04 & 0.11 & 2.48 \\
\hline $\mathrm{CD}(\mathrm{P}=0.05)$ & 0.38 & 0.12 & NS & 5.68 \\
\hline \multicolumn{5}{|l|}{ Nutrient levels (T) } \\
\hline $\mathbf{T}_{1}=20-10-15$ & 6.93 & 1.75 & 5.12 & 88.74 \\
\hline $\mathbf{T}_{2}=40-20-30$ & 7.53 & 1.90 & 5.22 & 99.45 \\
\hline $\mathbf{T}_{3}=60-30-45$ & 8.13 & 2.06 & 5.38 & 111.52 \\
\hline $\mathbf{T}_{4}=$ Vermicompost @ 2 t/ha & 7.05 & 1.78 & 5.48 & 97.62 \\
\hline $\mathbf{T}_{5}=20-10-15+$ Vermicompost @ $1 \mathrm{t} / \mathrm{ha}$ & 8.26 & 2.09 & 5.45 & 114.32 \\
\hline $\mathrm{T}_{6}=$ Control & 5.80 & 1.47 & 5.40 & 79.64 \\
\hline $\mathrm{SEm} \pm$ & 0.29 & 0.07 & 0.19 & 4.29 \\
\hline $\mathrm{CD}(\mathrm{P}=0.05)$ & 0.66 & 0.21 & NS & 9.82 \\
\hline Interaction $(\mathbf{S} \times \mathbf{T})$ & NS & NS & NS & NS \\
\hline
\end{tabular}


Table.5 Effect of different treatments on N, P, K- content

\begin{tabular}{|c|c|c|c|c|c|c|}
\hline \multirow[t]{2}{*}{ Treatments } & \multicolumn{2}{|c|}{ N- Content (\%) } & \multicolumn{2}{|c|}{ P-Content (\%) } & \multicolumn{2}{|c|}{ K- Content (\%) } \\
\hline & Leaf & Rhizome & Leaf & Rhizome & Leaf & Rhizome \\
\hline \multicolumn{7}{|l|}{ Crop Geometry (S) } \\
\hline $\mathrm{S}_{1}=20 \mathrm{~cm} \times 20 \mathrm{~cm}$ & 2.11 & 2.86 & 0.17 & 0.07 & 0.90 & 0.62 \\
\hline $\mathrm{S}_{2}=30 \mathrm{~cm} \times 30 \mathrm{~cm}$ & 2.29 & 2.90 & 0.16 & 0.06 & 0.89 & 0.61 \\
\hline $\mathrm{SEm} \pm$ & 0.07 & 0.05 & 0.004 & 0.001 & 0.02 & 0.02 \\
\hline $\mathrm{CD}(\mathrm{P}=0.05)$ & NS & NS & NS & NS & NS & NS \\
\hline \multicolumn{7}{|l|}{ Nutrient levels (T) } \\
\hline $\mathbf{T}_{1}=20-10-15$ & 2.13 & 2.87 & 0.16 & 0.07 & 0.87 & 0.60 \\
\hline $\mathbf{T}_{2}=40-20-30$ & 2.19 & 2.90 & 0.17 & 0.07 & 0.88 & 0.63 \\
\hline $\mathbf{T}_{3}=60-30-45$ & 2.23 & 2.90 & 0.17 & 0.07 & 0.94 & 0.63 \\
\hline $\mathbf{T}_{\mathbf{4}}=$ Vermicompost @ $2 \mathrm{t} / \mathrm{ha}$ & 2.18 & 2.88 & 0.16 & 0.07 & 0.88 & 0.62 \\
\hline $\begin{array}{l}\mathbf{T}_{\mathbf{5}}=20-10-15+\text { Vermicompost @ } 1 \\
\text { t/ha }\end{array}$ & 2.40 & 2.95 & 0.17 & 0.07 & 0.96 & 0.65 \\
\hline $\mathrm{T}_{6}=$ Control & 2.09 & 2.76 & 0.15 & 0.06 & 0.83 & 0.58 \\
\hline $\mathrm{SEm} \pm$ & 0.11 & 0.09 & 0.006 & 0.002 & 0.04 & 0.03 \\
\hline $\mathrm{CD}(\mathrm{P}=0.05)$ & NS & NS & NS & NS & NS & NS \\
\hline Interaction $(\mathbf{S} \times \mathbf{T})$ & $\mathbf{N S}$ & NS & NS & NS & NS & $\mathbf{N S}$ \\
\hline
\end{tabular}


Table.6 Effect of different treatments on N, P, K- uptake

\begin{tabular}{|c|c|c|c|c|c|c|c|c|c|}
\hline \multirow[t]{2}{*}{ Treatments } & \multicolumn{2}{|c|}{ N- Uptake (kg/ha) } & \multirow{2}{*}{$\begin{array}{l}\text { Total } \\
\text { uptake } \\
\text { (kg/ha }\end{array}$} & \multicolumn{2}{|c|}{ P-Uptake (kg/ha) } & \multirow{3}{*}{$\begin{array}{l}\text { Total } \\
\text { Uptake } \\
\text { (kg/ha) }\end{array}$} & \multicolumn{2}{|c|}{ K- Uptake (kg/ha) } & \multirow{3}{*}{$\begin{array}{l}\text { Total } \\
\text { Uptake } \\
\text { (kg/ha) }\end{array}$} \\
\hline & Leaf & Rhizome & & Leaf & Rhizome & & Leaf & Rhizome & \\
\hline \multicolumn{8}{|l|}{ Crop Geometry (S) } & & \\
\hline $\mathrm{S}_{1}=20 \mathrm{~cm} \mathrm{x} 20 \mathrm{~cm}$ & 23.98 & 61.39 & 85.36 & 1.89 & 1.43 & 3.33 & 10.29 & 13.5 & 23.80 \\
\hline $\mathrm{S}_{2}=30 \mathrm{~cm} \mathrm{x} 30 \mathrm{~cm}$ & 15.25 & 44.62 & 59.89 & 1.06 & 1.00 & 2.06 & 5.85 & 9.43 & 15.29 \\
\hline SEm & 0.53 & 0.92 & 1.08 & 0.03 & 0.02 & 0.06 & 0.19 & 0.30 & 0.50 \\
\hline $\mathrm{CD}(\mathrm{P}=0.05)$ & 1.55 & 2.69 & 3.15 & 1.14 & 0.07 & 0.19 & 0.55 & 0.88 & 1.46 \\
\hline \multicolumn{10}{|l|}{ Nutrient levels (T) } \\
\hline $\mathbf{T}_{1}=20-10-15$ & 18.07 & 49.59 & 67.66 & 1.39 & 1.16 & 2.55 & 7.54 & 10.63 & 18.17 \\
\hline$T_{2}=40-20-30$ & 19.95 & 55.00 & 74.95 & 1.54 & 1.27 & 2.82 & 8.12 & 12.01 & 20.13 \\
\hline$T_{3}=60-30-45$ & 22.19 & 59.76 & 82.02 & 1.67 & 1.37 & 3.05 & 9.51 & 13.14 & 22.65 \\
\hline $\begin{array}{l}\mathrm{T}_{4}=\text { Vermicompost } \\
\text { @ } 2 \mathrm{t} / \mathrm{ha}\end{array}$ & 18.28 & 51.49 & 69.76 & 1.43 & 1.19 & 2.63 & 7.58 & 11.10 & 18.68 \\
\hline $\begin{array}{l}\mathbf{T}_{\mathbf{5}}=20-10-15+ \\
\text { Vermicompost @ } 1 \\
\text { t/ha }\end{array}$ & 24.01 & 61.56 & 85.57 & 1.76 & 1.44 & 3.20 & 9.75 & 13.59 & 23.34 \\
\hline $\mathrm{T}_{6}=$ Control & 15.18 & 40.61 & 55.80 & 1.05 & 0.87 & 1.93 & 5.95 & 8.33 & 14.28 \\
\hline $\mathrm{SEm} \pm$ & 0.92 & 1.59 & 1.87 & 0.06 & 0.04 & 0.01 & 0.33 & 0.52 & 0.87 \\
\hline $\mathrm{CD}(\mathrm{P}=0.05)$ & 2.68 & 4.66 & 5.55 & 0.20 & 0.13 & 0.33 & 0.98 & 1.53 & 2.54 \\
\hline Interaction $(\mathbf{S} \times \mathbf{T})$ & NS & NS & NS & NS & NS & NS & NS & NS & NS \\
\hline
\end{tabular}


Table.7 Effect of different treatments on available N, P and K content, organic matter content, bulk density and pH of soil after harvest of crop

\begin{tabular}{|c|c|c|c|c|c|c|}
\hline \multirow[t]{2}{*}{ Treatments } & \multicolumn{3}{|c|}{ Available NPK (kg/ha) } & \multirow{2}{*}{$\begin{array}{c}\text { Organic } \\
\text { matter }(\%) \\
\end{array}$} & \multirow{2}{*}{$\begin{array}{l}\text { Bulk density } \\
\text { (g/ce) }\end{array}$} & \multirow[t]{2}{*}{$\mathbf{p H}$} \\
\hline & $\mathbf{N}$ & $\mathbf{P}_{2} \mathrm{O}_{5}$ & $\mathbf{K}_{2} \mathbf{O}$ & & & \\
\hline \multicolumn{7}{|l|}{ Crop Geometry (S) } \\
\hline $\mathrm{S}_{1}=20 \mathrm{~cm} \mathrm{x} 20 \mathrm{~cm}$ & 272.47 & 11.30 & 235.35 & 1.09 & 1.37 & 4.94 \\
\hline $\mathrm{S}_{2}=30 \mathrm{~cm} \mathrm{x} 30 \mathrm{~cm}$ & 272.8 & 11.29 & 235.95 & 1.09 & 1.37 & 4.94 \\
\hline SEm & 1.35 & 0.20 & 4.05 & 0.01 & 0.002 & 0.001 \\
\hline $\mathrm{CD}(\mathrm{P}=0.05)$ & NS & NS & NS & NS & NS & $\mathbf{N S}$ \\
\hline \multicolumn{7}{|l|}{ Nutrient levels (T) } \\
\hline $\mathbf{T}_{1}=20-10-15$ & 269.70 & 11.02 & 240.46 & 1.07 & 1.37 & 4.94 \\
\hline $\mathbf{T}_{2}=40-20-30$ & 271.79 & 11.18 & 241.36 & 1.08 & 1.37 & 4.94 \\
\hline$T_{3}=60-30-45$ & 273.88 & 11.48 & 241.36 & 1.09 & 1.37 & 4.94 \\
\hline $\mathbf{T}_{\mathbf{4}}=$ Vermicompost @ $2 \mathrm{t} / \mathrm{ha}$ & 275.97 & 11.35 & 231.44 & 1.11 & 1.36 & 4.93 \\
\hline $\begin{array}{l}\mathbf{T}_{\mathbf{5}}=20-10-15+\text { Vermicompost } \\
@ 1 \mathrm{t} / \mathrm{ha}\end{array}$ & 278.06 & 11.96 & 235.95 & 1.10 & 1.36 & 4.93 \\
\hline $\mathbf{T}_{6}=$ Control & 266.42 & 10.80 & 223.33 & 1.07 & 1.37 & 4.94 \\
\hline $\mathrm{SEm} \pm$ & 2.63 & 0.35 & 7.01 & 0.02 & 0.004 & 0.002 \\
\hline $\mathrm{CD}(\mathrm{P}=0.05)$ & NS & NS & NS & NS & $\mathrm{NS}$ & $\mathbf{N S}$ \\
\hline Interaction $(\mathrm{S} \times \mathrm{T})$ & NS & NS & NS & NS & NS & $\mathbf{N S}$ \\
\hline Initial value & 270.00 & 25.70 & 138.90 & 1.09 & 1.37 & 4.94 \\
\hline
\end{tabular}


Table.8 Effect of different treatments on monetary return of Acorus calamus

\begin{tabular}{|c|c|c|c|c|}
\hline Treatment & $\begin{array}{c}\text { Cost of } \\
\text { cultivation }\end{array}$ & Gross return & Net return & B:C ratio \\
\hline \multicolumn{5}{|l|}{ Crop Geometry (S) } \\
\hline $\mathrm{S}_{1}=20 \mathrm{~cm} \times 20 \mathrm{~cm}$ & 44898.00 & 107583.00 & 63685.00 & 1.42 \\
\hline$S_{2}=30 \mathrm{~cm} \times 30 \mathrm{~cm}$ & 34748.00 & 76750.00 & 43002.00 & 1.24 \\
\hline \multicolumn{5}{|l|}{ Nutrient levels (T) } \\
\hline $\mathbf{T}_{1}=20-10-15$ & 32233.00 & 87750.00 & 56517.00 & 1.75 \\
\hline $\mathbf{T}_{2}=40-20-30$ & 33405.00 & 95250.00 & 62845.00 & 1.88 \\
\hline $\mathbf{T}_{3}=60-30-45$ & 34577.00 & 102750.00 & 69173.00 & 2.00 \\
\hline $\mathbf{T}_{\mathbf{4}}=$ Vermicompost $(2 \mathrm{t} / \mathrm{ha})$ & 53405.00 & 89250.00 & 36845.00 & 0.69 \\
\hline $\mathbf{T}_{\mathbf{5}}=20-10-15+$ Vermicompost $(1 \mathrm{t} / \mathrm{ha})$ & 44577.00 & 104500.00 & 60923.00 & 1.37 \\
\hline $\mathrm{T}_{6}=$ Control & 33405.00 & 73500.00 & 41095.00 & 1.23 \\
\hline
\end{tabular}

Fig.1 Effect of crop geometry and nutrient levels on crop growth rate
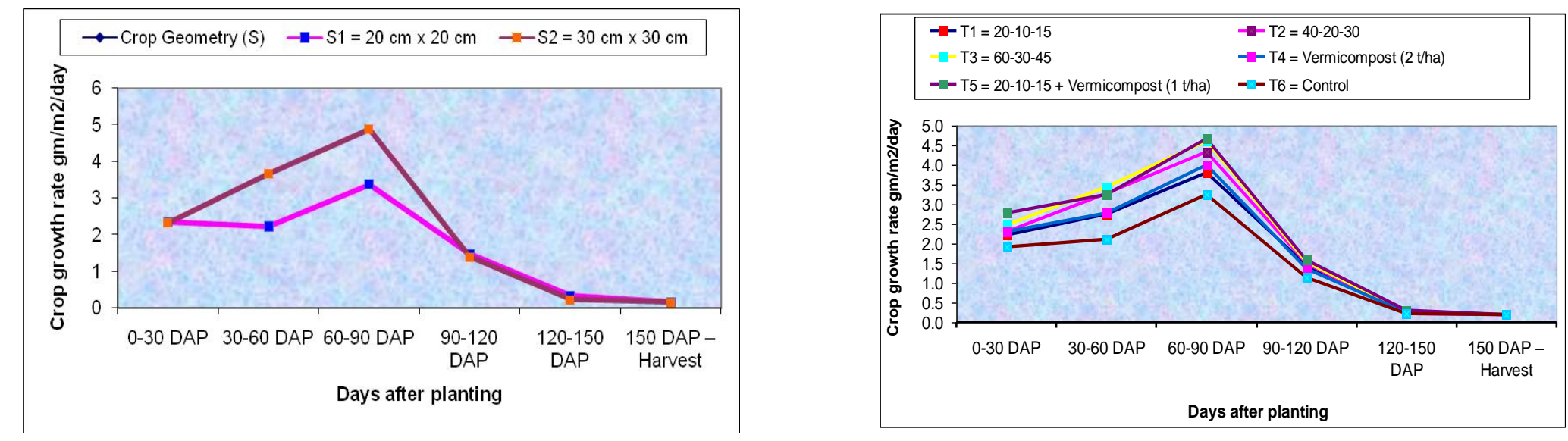
Among different nutrient management practices, higher yield attributing characters were observed under the application of organic and inorganic fertilizer i.e., 20-10$15 \mathrm{~kg} / \mathrm{ha} \mathrm{N}-\mathrm{P}_{2} \mathrm{O}_{5}-\mathrm{K}_{2} \mathrm{O}+$ Vermicompost (1t/ha) and was followed by application of 60-30$45 \mathrm{~kg} / \mathrm{ha} \quad \mathrm{N}-\mathrm{P}_{2} \mathrm{O}_{5}-\mathrm{K}_{2} \mathrm{O}$. The organic source enhanced the efficient utilization of native as well as added fertilizer nutrients, which maintained the balance between growth and yield attributes.Corroborative findings have been reported byDatta et al., (2009).

\section{Rhizome Yield}

The highest rhizome fresh and dry yield was recorded under crop spacing $20 \mathrm{~cm} \times 20 \mathrm{~cm}$. The increase in yield under closer spacing may be ascribed due to the higher plant density per unit area. Similar findings were reported by Singh and Nongmaithem (2013) and Tiwari et al., (2012) on Acoruscalmus and Bisen et al., (2007) in ginger. Present study also showed that rhizome yield increased significantly with application oforganic and inorganic fertilizer i.e., 20-10$15 \mathrm{~kg} / \mathrm{ha} \mathrm{N}-\mathrm{P}_{2} \mathrm{O}_{5}-\mathrm{K}_{2} \mathrm{O}+$ Vermicompost (1t/ha) and was followed by application of 60-30-45 $\mathrm{kg} / \mathrm{ha} \quad \mathrm{N}-\mathrm{P}_{2} \mathrm{O}_{5}-\mathrm{K}_{2} \mathrm{O}$. Higher rhizome yield with the combined application of organic and inorganic fertilizer might be attributed to higher values of various yield attributing characters under this treatment. The treatments which resulted in higher values of yield attributing characters also resulted in higher rhizome yield of Acorus calamus. Similar result was reported by Datta et al., (2009). Increased in yield due to application of organic manures have been reported by Vadiraj et al., (1998) in turmeric and Paturade et al., (2002) in Safedmusli.

\section{Rhizome oil content and oil yield}

Different crop geometry could not bring about any significant influence in percent oil content of Acorus calamus. However the oil yield was found to be significantly higher under closer crop spacing $20 \mathrm{~cm} \times 20 \mathrm{~cm}$. Higher oil yield under closer spacing might be due to higher rhizome yield resulting more oil yield. Corroborative findings have been reported by Singhand Nongmaithem (2013).

Oil content in rhizome was non-significant under different nutrient management practices. But the oil yield was found to higher under the integrated nutrient management practice i.e., application of 20$10-15 \mathrm{~kg} /$ ha $\mathrm{N}-\mathrm{P}_{2} \mathrm{O}_{5}-\mathrm{K}_{2} \mathrm{O}+$ Vermicompost (1t/ha) and was follwed by application of 60$30-45 \mathrm{~kg} / \mathrm{ha} \quad \mathrm{N}-\mathrm{P}_{2} \mathrm{O}_{5}-\mathrm{K}_{2} \mathrm{O}$. Higher rhizome production due to the availability of both macro and micro nutrients under the integrated nutrient management practice resulted in higher rhizome yield. Similar results have been reported by Datta et al., (2009).

\section{Nutrient content and uptake}

$\mathrm{N}, \mathrm{P}$ and $\mathrm{K}$-content in leaf and rhizome of Acorus calamus did not vary significantly under different crop spacings. However, closer crop spacing resulted in significantly higher N, P and K-uptake by leaf and rhizome than wider spacing. This difference in nutrient uptake by Acorus calamus might be explained on the basis of differences in biomass (both leaf and rhizome) brought about by crop spacings. Higher degree of competition due to accomodation of higher number of plants under closer spacing might have resulted in higher uptake. Similar results have been reported by Ganorkar et al., (2006) in Safedmusli.

Different nutrient management practices did not bring about any significant change in nutrient content in leaf and rhizome of Acorus calamus. However highest N, P and K-uptake by Acorus calamus was observed under 
application of $20-10-15 \mathrm{~kg} / \mathrm{ha} \mathrm{N}-\mathrm{P}_{2} \mathrm{O}_{5}-\mathrm{K}_{2} \mathrm{O}+$ Vermicompost (1t/ha). Such results are obvious as application of organic and inorganic nutrients improved the total biomass production and thereby increased the uptake of $\mathrm{N}, \mathrm{P}$ and $\mathrm{K}$.

Available soil $\mathrm{N}, \mathrm{P}_{2} \mathrm{O}_{5}$ and $\mathrm{K}_{2} \mathrm{O}$, soil organic matter, soil bulk density and soil $\mathrm{pH}$ were not significantly influenced by crop spacing and nutrient levels.

\section{Economics}

A perusal of data indicated that the highest gross return of 107583.00 per hectare was obtained at crop spacing at $20 \mathrm{~cm} \times 20 \mathrm{cmand}$ was followed by crop spacing at $30 \mathrm{~cm} \times 30$ $\mathrm{cm}$. Crop spacing of $20 \mathrm{~cm} \times 20 \mathrm{~cm}$ also recorded the highest net return ( 63685.00 ) and $\mathrm{B}$ : $\mathrm{C}$ ratio (1.42) and followed by crop spacing of $30 \mathrm{~cm} \times 30 \mathrm{~cm}$ ( 43002.00$)$. Under different nutrient management practices, highest gross return of 104500.00 per hectare was received under application of both organic and inorganic fertilizers i.e., 2010-15 kg/ha N-P $\mathrm{O}_{5}-\mathrm{K}_{2} \mathrm{O}+$ Vermicompost @ 1 t/ha. This was closely followed by application of $60-30-45 \mathrm{~kg} / \mathrm{ha} \mathrm{N}-\mathrm{P}_{2} \mathrm{O}_{5}-\mathrm{K}_{2} \mathrm{O}$ ( 102750.00). The highest net return ( 69173.00) was obtained fromapplication of 60-30-45 kg/ha N-P $\mathrm{O}_{5}-\mathrm{K}_{2} \mathrm{O}$ and this was followed by application of 20-10-15 kg/ha N$\mathrm{P}_{2} \mathrm{O}_{5}-\mathrm{K}_{2} \mathrm{O}+$ Vermicompost @ 1 t/ha ( 60923.00). The increase in gross and net return might be due to positive effect of these treatments on growth and yield attributes resulting in higher rhizome yield.

Based on one year of field experiment and laboratory studies it could be concluded that Acorus calamus should be planted at $20 \mathrm{~cm} \times$ $20 \mathrm{~cm}$ spacing with a fertilizer dose 60-30-45 $\mathrm{kg} / \mathrm{ha} \quad \mathrm{N}-\mathrm{P}_{2} \mathrm{O}_{5}-\mathrm{K}_{2} \mathrm{O}$ or $20-10-15 \mathrm{~kg} / \mathrm{ha} \mathrm{N}$ $\mathrm{P}_{2} \mathrm{O}_{5}-\mathrm{K}_{2} \mathrm{O}+$ Vermicompost @ 1 t/ha to get higher rhizome yield and economic return.

\section{References}

Abrha, H.; Belew, D. and woldegiorgis, G. (2014). Effect of inter and intra row spacing on tuber yield and yield components of potato (Solanum tuberosum L.) at oflaworeda, Northern Ethiopia. African Journal of Plant Science 8(6): 285-290.

Bhaskar, S.; Kumar, T.V.; Shivananda, T.N.; Arun, M.N.; Janardan, G. and Ramchandra, C. (2001). Effect of farmyard manure, nitrogen levels and its method of application on scented geranium (Pelargonium graveolens). Journal of Medicinal and Aromatic plants 23(3): 388-391.

Bhuvaneswari, R. (2012). Anti-bacterial activity of Acorus calamus and some derivatives against fish pathogen Aeromonas hydrophila, Indian Journal of Fundamental and Applied Life Science 2(2): 191-201

Bisen, R.k.; Singh, P.R.; Agarwal, H.P. and Sahu, A.K. (2007).Response of nitrogen, phosphorus and spacing on production in ginger (Zingiber officinalroes). Journal of Eco-Friendly Agriculture 2(2): 126-127.

Datta, S.; Dey, A. N. And Maitra, S. (2009). Effect of FYM and $\mathrm{GA}_{3}$ on growth and yield, of Sweet Flag (Acorus calamus L.) under Terai zone of West Bengal, Journal of Horticltural Science 4(1): 59-62.

Devi, S. A. and Ganjewala, D. (2009). Antimicrobial activity of Acorus calamus (L) rhizome and leaf extract, Acta Biologica Szegediensis 53(1): 45-49.

Gaidhani, S.N.; Lavekar, G.S.; Juvekar, A.S.; Sen, S.; Singh A. and Kumar, S. (2009). In vitro anti-cancer activity of standard extracts used in Ayurveda, Pharmacog. Mag 5(20): 425429

Ganokar, S.R.; Barabde, N.P.; Futane, N.W.: Pote, S.R. and Wankhade, S.G. (2006). Nutrient upake by Safedmusli (Chlorophytum borivilanum) as influnenced by different levels of FYM and nitrogen. Journal of soils and crops 16 (1): 61-68.

Jackson, M.L. (1973). In: Soil chemical analysis. Pub. Prentica Hall of India Pvt. Ltd., New Delhi.

Khanna, S.S. and Yadav, D.V. (1979). In: Practical manual for introductory courses in soil. Pub. Director of Pub. HAV, Hisar. 
Kima, H.;Hanb, T.H. and Lee, S.G. (2009) Antiinflammatory activity of a water extract of Acorus calamus L. leaves on keratinocyteHaCaT cells, Journal of enthanopharmocology, 122: 149-156

McAlpine T. \& Warrier Limited (1996). Future World Trends in the Supply, Utilisation, and Marketing of Endangered Plant Species.

Mishra, N.K. (2013). Impact of fly-ash amended soil on growth and rhizome setting in an endangered medicinal plant Acorus calamus Linn., Indian Journal of Life Science2(2): 99-102.

Muthuraman, A. and Singh, N. (2012). Attenuating effect of Acorus calamus extract in chronic constriction injury induced neuropathic pain in rats: an evidence of antioxidative, anti-inflammatory, neuroprotective and calcium inhibitory effects, BMC complement. Altern. Med.11: 24-37.

Paturade, J.T.; Wankhade, S.G.; Khode, P.P.; Chatol, P.U.; Deo, D.D. and Bhayur, S.A. (2002). Effect of organic manures and plant population on yield of Safedmusli (Chloraphytum borivallianum). Agricultural Science Digest 22(1): 51-52.

Phongpaichit, S.; Pujenjob, N.; Rukachaisirikul, V. and Ongsakul, M. (2005). Antimicrobial activities of the crude methanol extract of Acorus calamus Linn., Songklanakarin J. Sci. Technol 27: 517-523.

Singh, M.C.; Kumar, A. and Kishore, N. (2010).Effect of different organic manures and spacing on Aloe vera. Annals of Agril. Res. New Series 31(3 and 4): 107-110.

Singh, M.S. and Nongmaithe, D. (2013). Growth attributes and rhizome yield of Sweet flag (Acorus calamus L.) as influenced by spacing, The Bioscan 8(4): 2007-2009

Singh. K.K.; Tiwari, R.S. and Agarwal, A. (2003). Response of organic and inorganic fertilizers in quality improvement in garlic. Souvienir and Abstract, National Symposium on Organic Farming in Horticulture for Sustainable Production 58: 29-30.

Tiwari, J. P.; Tiwari, A. B. and Tiwari, G. (2000). Effect of nitrogen application on growth and yield of Acorus calamus, Journal of Medicinal and Aromatic plant sciences 22: 636-638.

Tiwari, R. K. S.; Das, K.; Pandey, D.; Tiwari, R. B. and Dubey J. (2012). Rhizome Yield of Sweet Flag (Acorus calamus L.) as Influenced by Planting Season, Harvest Time, and Spacing, International Journal of Agronomy. 2012, Article ID 731375, 8 pages doi:10.1155/2012/731375

Tiwari, R.K.S.; Shambharkar, V.B.; Chaure N.K. and Thakur, B.S. (2004). Prospect of cultivation of Acorus calamus (Bach) under rainfed rice ecosystem. In Proceedings of the Abstracts of International Symposium on Rainfed Rice Ecosystems: Perspective and Potential, 161-162.

Vadiraj, B.A.; Siddagangaiah, S. and Porti, N. (1998). Effect of vermicompost on the growth and yield of turmeric. South Indian Horticulture 46(3-6): 176-179.

Vojtiskova, L.; Munzarova, E.; Votrubova, O.; Rihova, A. and Juricova, B. (2004). Growth and biomass allocation of sweet flag (Acorus calamus L.) under different nutrient conditions, Hydrobiologia 518: 9-22.

Walkely, A. and Black, C.A. (1934). An experimentation of the method for determination of soil organic matter and proposed modification of the chromic acid titartion method. Soil science 37: 29-39.

Watson, D.J. (1952). The physiological basis of variation in yield. Adv. Agronomy 4: 101144.

Wu, H.S.; Zhu, D.F. and Zhou, C. (2009). Insulin sensitizing activity of ethyl acetate fraction of Acorus calamus L. in vitro and in vivo, Journal Ethnopharmacol. 123(2): 288-92.

\section{How to cite this article:}

Payeng C., A. Sharma and Gogoi B. 2018. Effect of Nutrient Management and Crop Geometry in Sweet Flag. Int.J.Curr.Microbiol.App.Sci. 7(06): 2678-2693.

doi: https://doi.org/10.20546/ijcmas.2018.706.317 